\title{
On the Expressivity of Feature Logics with Negation, Functional Uncertainty, and Sort Equations
}

Franz Baader, Hans-Jürgen Bürckert, Bernhard Nebel, Werner Nutt, and Gert Smolka

$$
\text { January } 1991
$$

Deutsches Forschungszentrum für Künstliche Intelligenz $\mathrm{GmbH}$

Postfach 2080

D-6750 Kaiserslautern, FRG

Tel.: (+49 631) 205-3211/13

Fax: (+49 631) 205-3210
Stuhlsatzenhausweg 3

D-6600 Saarbrücken 11, FRG

Tel.: (+49 681) 302-5252

Fax: (+49 681) 302-5341 


\section{Deutsches Forschungszentrum für Künstliche Intelligenz}

The German Research Center for Artificial Intelligence (Deutsches Forschungszentrum für Künstliche Intelligenz, DFKI) with sites in Kaiserslautern und Saarbrücken is a non-profit organization which was founded in 1988 by the shareholder companies ADV/Orga, AEG, IBM, Insiders, Fraunhofer Gesellschaft, GMD, Krupp-Atlas, Mannesmann-Kienzle, Philips, Siemens and Siemens-Nixdorf. Research projects conducted at the DFKI are funded by the German Ministry for Research and Technology, by the shareholder companies, or by other industrial contracts.

The DFKI conducts application-oriented basic research in the field of artificial intelligence and other related subfields of computer science. The overall goal is to construct systems with technical knowledge and common sense which - by using Al methods - implement a problem solution for a selected application area. Currently, there are the following research areas at the DFKI:

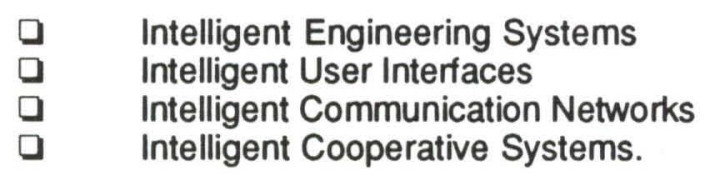

The DFKI strives at making its research results available to the scientific community. There exist many contacts to domestic and foreign research institutions, both in academy and industry. The DFKI hosts technology transfer workshops for shareholders and other interested groups in order to inform about the current state of research.

From its beginning, the DFKI has provided an attractive working environment for $\mathrm{Al}$ researchers from Germany and from all over the world. The goal is to have a staff of about 100 researchers at the end of the building-up phase.

Prof. Dr. Gerhard Barth

Director 
On the Expressivity of Feature Logics with Negation, Functional Uncertainty, and Sort Equations

Franz Baader, Hans-Jürgen Bürckert, Bernhard Nebel, Werner Nutt, and Gert Smolka

DFKI-RR-91-01 
(C) Deutsches Forschungszentrum für Künstliche Intelligenz 1990

This work may not be copied or reproduced in whole or in part for any commercial purpose. Permission to copy in whole or in part without payment of fee is granted for nonprofit educational and research purposes provided that all such whole or partial copies include the following: a notice that such copying is by permission of Deutsches Forschungszentrum für Künstliche Intelligenz, Kaiserslautern, Federal Republic of Germany; an acknowledgement of the authors and individual contributors to the work; all applicable portions of this copyright notice. Copying, reproducing, or republishing for any other purpose shall require a licence with payment of fee to Deutsches Forschungszentrum für Künstliche Intelligenz. 


\title{
On the Expressivity of Feature Logics with Negation, Functional Uncertainty, and Sort Equations
}

\author{
Franz Baader, Hans-Jürgen Bürckert, Bernhard Nebel, \\ Werner Nutt, and Gert Smolka \\ German Research Center for Artificial Intelligence \\ Kaiserslautern and Saarbrücken, Germany
}

\begin{abstract}
Feature logics are the logical basis for so-called unification grammars studied in computational linguistics. We investigate the expressivity of feature terms with negation and the functional uncertainty construct needed for the description of long-distance dependencies and obtain the following results: satisfiability of feature terms is undecidable, sort equations can be internalized, consistency of sort equations is decidable if there is at least one atom, and consistency of sort equations is undecidable if there is no atom.
\end{abstract}




\section{Contents}

1 Introduction $\quad 3$

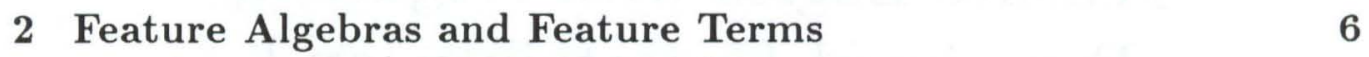

3 Rooted Feature Algebras $\quad 9$

4 Internalizing Sort Equations $\quad 12$

5 Consistency of Sort Equations $\quad 14$

$\begin{array}{lll}6 & \text { Satisfiability is Undecidable } & 17\end{array}$

$\begin{array}{lll}7 \text { Conclusion } & 18\end{array}$ 


\section{Introduction}

Feature constraint grammars, also known as unification grammars, have become the predominant family of declarative grammar formalisms in Computational Linguistics $[10,6,18,17,14]$. The common assumption of these formalisms is that linguistic objects can be described by means of their features, which are functional attributes. Figure 1, for instance, shows the description of a linguistic object that may represent the sentence "John sings a song". The features appear as edges of the graph. The terminal nodes are atoms representing primitive linguistic objects.

Kasper and Rounds $[9,15]$ were the first to capture the relation between feature descriptions and linguistics objects in terms of a logic. Subsequently, Johnson [5] and Smolka [19, 20] realized that feature logics can be modeled straightforwardly in Predicate Logic. In this approach, which underlies the present paper, a domain of linguistic objects is called a feature algebra and is simply a structure that interprets atoms as pairwise distinct individuals and features as unary partial functions that are undefined on atoms. In addition, one can have sorts, which are interpreted as sets of individuals. Dörre and Rounds [4] relate feature algebras to term algebras und show the undecidability of the semiunification problem.

One popular syntax for feature descriptions are so-called feature terms $[9,15,20]$, which are expressions denoting sets in feature algebras. The basic feature term forms are given by

$$
S \longrightarrow a|A| p: S|p \downarrow q| S \sqcap S^{\prime}\left|S \sqcup S^{\prime}\right| \neg S,
$$

where $a$ stands for atoms, $A$ stands for sorts, and $p$ and $q$ stand for words over features. Given a feature algebra, $a$ denotes the singleton consisting of the atom $a, p$ : $S$ denotes the inverse image of $S$ under $p$ (where $p$ is interpreted as unary partial function obtained as the composition of its features), $p \downarrow q$ denotes the set of all individuals for which $p$ and $q$ are defined and agree, $S \sqcap S^{\prime}$ denotes the intersection of $S$ and $S^{\prime}, S \sqcup S^{\prime}$ denotes the union of $S$ and $S^{\prime}$, and $\neg S$ denotes the complement of $S$. For applications it is important to decide whether a feature term is satisfiable, that is, whether it denotes a nonempty set in some feature algebra. The satisfiability problem for feature terms as given above is NP-complete [20].

A sort equation is a pair $S \doteq S^{\prime}$ consisting of two feature terms. A feature algebra is a model of a set of sort equations if for every equation both sides denote the same set. 


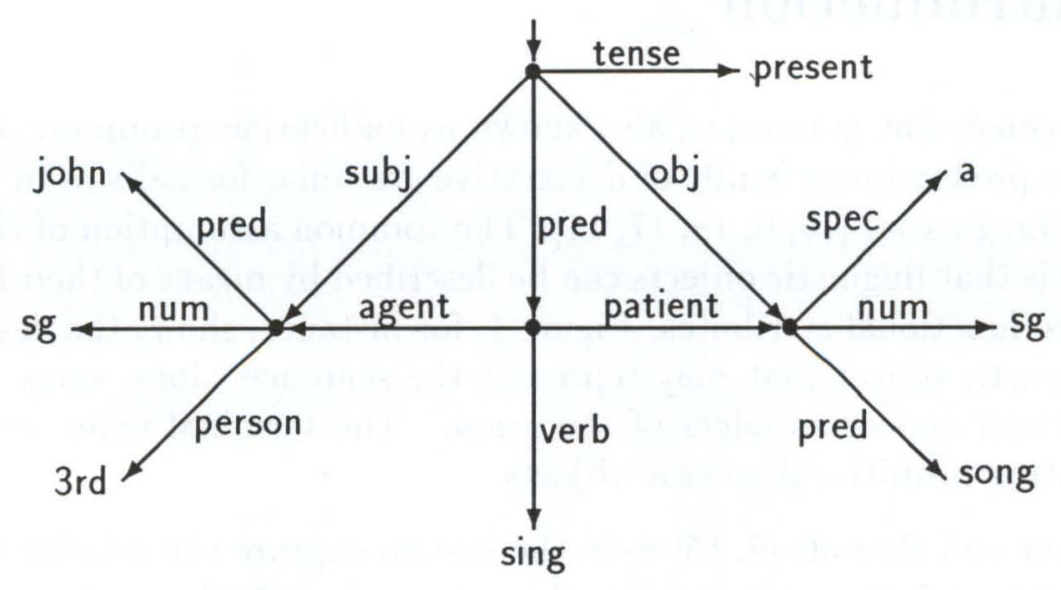

Figure 1: A feature graph.

Grammar rules in Functional Unification Grammar [10] and the more recent HPSG [14] are stated by means of sort equations. Figure 2 shows a simple grammar in this style (sorts start with capital letters), which generates the single sentence "John sings a song", provided the right assumptions on word order are made. The basic idea is that in a model of the grammar the elements of a sort are the linguistic objects of the syntactic category expressed by the sort. Note that the graph in Figure 1 describes an element of the sort $\mathrm{S}$ in some model of the grammar in Figure 2.

In this paper we investigate the expressivity of an additional feature term form that is known as functional uncertainty $[7,8]$ and was invented for the convenient description of so-called long-distance dependencies in the grammar formalism LFG [6]. It takes the form

$$
\exists L(S),
$$

where $L$ is a finite description of a regular set of words over features and $S$ is a feature term. A feature term $\exists L(S)$ denotes the set of all individuals $d$ such that there exists a word $p \in L$ such that $d$ is in the inverse image of $S$ under $p$. One can think of $\exists L(S)$ as the possibly infinite union

$$
p_{1}: S \sqcup p_{2}: S \sqcup p_{3}: S \sqcup \cdots,
$$

where $p_{1}, p_{2}, p_{3}, \ldots$ are the words in $L$. Note that the form $p: S$ can be expressed with $\exists L(S)$ if one takes for $L$ the singleton consisting of the word $p$. 


$$
\begin{aligned}
& \mathrm{S} \doteq(\text { subj: NP) } \sqcap \mathrm{VP} \\
& N P \doteq(D \sqcap N) \sqcup \text { Name } \\
& V P \doteq V \sqcap(\text { obj: NP) } \\
& D \doteq \text { (num:sg) } \sqcap \text { (spec: } a) \\
& \mathrm{N} \doteq \text { (num:sg) } \sqcap \text { (pred: song) } \\
& \text { Name } \doteq \text { (num: } \mathrm{sg}) \sqcap(\text { person: } 3 r d) \sqcap(\text { pred: john }) \\
& \vee \doteq \text { (subj num: } s g) \sqcap \text { (subj person: } 3 \text { rd) } \sqcap \text { (pred verb: sing) } \Pi \\
& \text { (tense: present) } \sqcap \text { (pred agent } \downarrow \text { subj) } \sqcap \text { (pred patient } \downarrow \text { object) }
\end{aligned}
$$

Figure 2: A simple grammar.

So far, the decidability of the satisfiability of feature terms with functional uncertainty has been an open problem. In this paper we show that it is undecidable, even if there are no atoms. However, our result depends crucially on the presence of the negation $\neg S$. Thus the problem is still open if only feature terms built with the forms $a, p \downarrow q, S \sqcap S^{\prime}$, and $\exists L(S)$ are considered. This restricted problem is addressed in [7], where a partial solution involving an acyclicity condition is given.

There is a surprising connection between functional uncertainty and sort equations. We will exhibit an algorithm that, given a finite set $\mathcal{E}$ of sort equations and a feature term $S$, produces finitely many feature terms $S_{1}, \ldots, S_{n}$ such that $S$ is satisfiable in a model of $\mathcal{E}$ if and only if $S_{1}, \ldots, S_{n}$ are satisfiable in some (arbitrary) feature algebra. This result says that, in the presence of functional uncertainty and negation, sort equations can be internalized and thus do not yield additional expressivity with respect to satisfiability. Since it is known that satisfiability with respect to sort equations is undecidable [20], this result immediately implies that satisfiability of feature terms with functional uncertainty and negation is undecidable.

As an interesting byproduct of the internalization result for sort equations, we will show that, somewhat surprisingly, it is decidable whether a finite set of sort equations has a model, provided there is at least one atom. However, if we do not assume atoms, the consistency of sort equations becomes undecidable, even if we disallow feature terms with functional uncertainty.

The paper is organized as follows. Section 2 defines feature algebras, feature terms and sort equations and states basic properties. Section 3 shows 
that to decide satisfiability of feature terms it suffices to consider only the roots of rooted feature algebras, an auxiliary result on which the rest of the paper depends. Section 4 shows how sort equations can be expressed with functional uncertainty (our first main result). Section 5 shows that consistency of sort equations is decidable if there is at least one atom while it is undecidable if there is no atom (our second main result). Section 6 shows that satisfiability of feature terms with functional uncertainty and negation is undecidable (our third main result). Section 7 concludes.

\section{Feature Algebras and Feature Terms}

We assume three pairwise disjoint, possibly empty sets of symbols: atoms (denoted by $a, b, c$ ), sorts (denoted by $A, B, C$ ), and features (denoted by $f, g, h)$. In the following, let $\mathbf{A}$ denote the set of all atoms, $\mathbf{S}$ the set of all sorts, and $\mathbf{F}$ the set of all features. We assume that there is at least one symbol, that is, $\mathbf{A} \cup \mathbf{S} \cup \mathbf{F} \neq \emptyset$.

A feature algebra is a pair $\left(\mathbf{D}^{\mathcal{I}}, \cdot^{\mathcal{I}}\right)$ consisting of a nonempty set $\mathbf{D}^{\mathcal{I}}$ (the domain of $\mathcal{I}$ ) and an interpretation function ${ }^{\mathcal{I}}$ assigning to every atom $a$ an element $a^{\mathcal{I}} \in \mathbf{D}^{\mathcal{I}}$, to every sort $A$ a subset $A^{\mathcal{I}} \subseteq \mathbf{D}^{\mathcal{I}}$, and to every feature $f$ a set of ordered pairs $f^{\mathcal{I}} \subseteq \mathbf{D}^{\mathcal{I}} \times \mathbf{D}^{\mathcal{I}}$ such that the following conditions are satisfied:

1. if $(d, e)$ and $\left(d, e^{\prime}\right)$ are in $f^{\mathcal{T}}$, then $e=e^{\prime}$ (features are functional)

2. if $a \neq b$, then $a^{\mathcal{I}} \neq b^{\mathcal{I}}$ (unique name assumption)

3. if $f$ is a feature and $a$ is an atom, then there exists no $d \in \mathbf{D}^{\mathcal{I}}$ such that $\left(a^{\mathcal{I}}, d\right) \in f^{\mathcal{I}}$ (atoms are primitive).

Note that we can see features equivalently either as functional binary relations or as unary partial functions. In place of $(d, e) \in f^{\mathcal{I}}$ we shall equivalently use the notation $e=d f^{\mathcal{I}}$, which means that the partial function $f^{\mathcal{I}}$, if applied to $d$, yields the value $e$. If there exists no $e$ such that $(d, e) \in$ $f^{\mathcal{I}}$ we say that $d f^{\mathcal{I}}$ is undefined. We use suffix notation for application of partial functions because we want to write composition of binary relations and partial functions from left to right, that is, $f^{\mathcal{I}} g^{\mathcal{I}}$ will mean apply first $f^{\mathcal{I}}$ and then $g^{\mathcal{I}}$.

A path is a word in $\mathbf{F}^{*}$, that is, a finite, possibly empty sequence of features. We shall use the letters $p, q$, and $r$ for paths. Let $\mathcal{I}$ be a feature 
algebra and $p=f_{1} \cdots f_{n}(n \geq 0)$ be a path. The empty path $\varepsilon$ is interpreted as the identity on $\mathbf{D}^{\mathcal{I}}$. For $n \geq 1, p$ is interpreted as the functional binary relation $p^{\mathcal{I}}$ which is obtained by composition of the functional binary relations $f_{1}^{I}, \ldots, f_{n}^{I}$, that is,

$$
\begin{aligned}
(d, e) \in\left(f_{1} \cdots f_{n}\right)^{\mathcal{I}} \Longleftrightarrow \quad \exists d_{0}, & \ldots, d_{n}: d=d_{0} \wedge d_{n}=e \\
& \wedge\left(d_{0}, d_{1}\right) \in f_{1}^{\mathcal{I}} \wedge \ldots \wedge\left(d_{n-1}, d_{n}\right) \in f_{n}^{\mathcal{I}} .
\end{aligned}
$$

As for single features, we shall often write $e=d\left(f_{1} \cdots f_{n}\right)^{\mathcal{I}}$ instead of $(d, e) \in$ $\left(f_{1} \cdots f_{n}\right)^{\mathcal{I}}$, and shall say $d\left(f_{1} \cdots f_{n}\right)^{\mathcal{I}}$ is undefined if there exists no such $e$.

Regular sets of paths can be specified by finite means, for instance, by regular expressions over the alphabet of all features. The letter $L$ will always denote a finite description of a regular set of paths, and we write $p \in L$ if the path $p$ is in the regular set specified by $L$. As usual, we shall take $\emptyset$ as description of the empty set of paths, and for a path, $p$ as description of the singleton $\{p\}$.

Feature terms are descriptions that denote sets in feature algebras. Here is the abstract syntax of feature terms:

$\begin{array}{rll}S, T \longrightarrow & a & \text { atom } \\ A & \text { sort } \\ p: S & \text { selection } \\ p \downarrow q & \text { agreement } \\ \perp & \text { bottom } \\ \top & \text { top } \\ S \sqcap T & \text { intersection } \\ S \sqcup T & \text { union } \\ \neg S & \text { negation } \\ S-T & \text { difference } \\ \exists L(S) & \text { existential path quantification } \\ & \forall L(S) & \text { universal path quantification. }\end{array}$

Because of the symmetry with universal path quantification we prefer to call the functional uncertainty construct existential path quantification. We will see that universal path quantification can be expressed with existential path quantification and negation.

It is important to note that our feature term language is parameterized with respect to the three alphabets of atoms, sorts and features, and that each of these alphabets may be empty. 
Given a feature algebra $\mathcal{I}$, the denotation $S^{\mathcal{I}}$ of a feature term $S$ in $\mathcal{I}$ is a subset of $\mathbf{D}^{\mathcal{I}}$ defined inductively as follows:

$$
\begin{aligned}
(a)^{\mathcal{I}} & =\left\{a^{\mathcal{I}}\right\} \\
(p: S)^{\mathcal{I}} & =\left\{d \in \mathbf{D}^{\mathcal{I}} \mid \exists e \in S^{\mathcal{I}}:(d, e) \in p^{\mathcal{I}}\right\} \\
(p \downarrow q)^{\mathcal{I}} & =\left\{d \in \mathbf{D}^{\mathcal{I}} \mid \exists e \in \mathbf{D}^{\mathcal{I}}:(d, e) \in p^{\mathcal{I}} \cap q^{\mathcal{I}}\right\} \\
\perp^{\mathcal{I}} & =\emptyset \\
\mathrm{T}^{\mathcal{I}} & =\mathbf{D}^{\mathcal{I}} \\
(S \sqcap T)^{\mathcal{I}} & =S^{\mathcal{I}} \cap T^{\mathcal{I}} \\
(S \sqcup T)^{\mathcal{I}} & =S^{\mathcal{I}} \cup T^{\mathcal{I}} \\
(\neg S)^{\mathcal{I}} & =\mathbf{D}^{\mathcal{I}}-S^{\mathcal{I}} \\
(S-T)^{\mathcal{I}} & =S^{\mathcal{I}}-T^{\mathcal{I}} \\
(\exists L(S))^{\mathcal{I}} & =\left\{d \in \mathbf{D}^{\mathcal{I}} \mid \exists p \in L \exists(d, e) \in p^{\mathcal{I}}: e \in S^{\mathcal{I}}\right\} \\
(\forall L(S))^{\mathcal{I}} & =\left\{d \in \mathbf{D}^{\mathcal{I}} \mid \forall p \in L \forall(d, e) \in p^{\mathcal{I}}: e \in S^{\mathcal{I}}\right\} .
\end{aligned}
$$

Note that if a feature term $S$ is a sort, $S^{\mathcal{I}}$ is given directly by the feature algebra $\mathcal{I}$.

Two feature terms $S$ and $T$ are equivalent (written $S \sim T$ ) if $S^{\mathcal{I}}=T^{\mathcal{I}}$ for every feature algebra $\mathcal{I}$.

Many of the introduced feature term forms are redundant. By rewriting with the equivalences

$$
\begin{aligned}
p: S & \sim \exists p(S) \\
\forall L(S) & \sim \neg \exists L(\neg S) \\
\perp & \sim \exists \emptyset(S) \quad \text { (where } S \text { is an arbitrary feature term) } \\
\top & \sim \forall \emptyset(S) \quad \text { (where } S \text { is an arbitrary feature term) } \\
S \sqcup T & \sim \neg(\neg S \sqcap \neg T) \\
S-T & \sim S \sqcap \neg T
\end{aligned}
$$

the forms appearing as the left hand sides can be eliminated. Obviously, the equivalences for $T$ and $\perp$ can only be used to eliminate these forms if there exists a feature term $S$ containing neither $T$ nor $\perp$. This is in fact the case since we assumed $\mathbf{A} \cup \mathbf{S} \cup \mathbf{F}$ to be nonempty.

Proposition 2.1 For every feature term one can compute in linear time an equivalent feature term containing only the forms a, $A, p \downarrow q, \exists L(S), S \sqcap T$, and $\neg S$. 
A feature term $S$ is called satisfiable if there exists a feature algebra $\mathcal{I}$ such that $S^{\mathcal{I}} \neq \emptyset$. Due to the presence of negation, unsatisfiability and equivalence of feature terms are linear-time reducible to each other:

$$
\begin{aligned}
S \text { unsatisfiable } & \Longleftrightarrow S \sim \perp \\
S \sim T & \Longleftrightarrow(S-T) \sqcup(T-S) \text { unsatisfiable. }
\end{aligned}
$$

Until now we have defined satisfiability, equivalence and inclusion of feature terms with respect to all feature algebras. One can also use axioms to specify classes of feature algebras with respect to which satisfiability, equivalence and inclusion should be considered. As axioms we use so-called sort equations which take the form $S \doteq T$, where $S$ and $T$ are feature terms. A feature algebra $\mathcal{I}$ satisfies a sort equation $S \doteq T$ iff $S^{\mathcal{I}}=T^{\mathcal{I}}$. A feature algebra $\mathcal{I}$ is a model of a set $\mathcal{E}$ of sort equations iff it satisfies every sort equation in $\mathcal{E}$. A set of sort equations is called consistent iff it has at least one model. We say that a feature term $S$ is satisfiable w.r.t. a set $\mathcal{E}$ of sort equations iff there exists a model $\mathcal{I}$ of $\mathcal{E}$ such that $\mathcal{S}^{\mathcal{I}} \neq \emptyset$. As for the case without sort equations, unsatisfiability, inclusion, and equivalence of feature terms w.r.t. a set of sort equations are linear-time reducible to each other.

Finitely many sort equations can always be equivalently expressed by a single sort equation of the form $S \doteq \perp$. In fact, a feature algebra $\mathcal{I}$ satisfies a sort equation $S \doteq T$ iff it satisfies $(S-T) \sqcup(T-S) \doteq \perp$; and $\mathcal{I}$ satisfies the sort equations $S_{1} \doteq \perp, \ldots, S_{n} \doteq \perp$ iff it satisfies $S_{1} \sqcup \ldots \sqcup S_{n} \doteq \perp$.

\section{Rooted Feature Algebras}

Let $S$ be a satisfiable feature term, and let the feature algebra $\mathcal{I}$ together with the element $d \in \mathbf{D}^{\mathcal{I}}$ be a witness for the satisfiability of $S$, that is, let $d \in S^{\mathcal{I}}$. Then $\mathbf{D}^{\mathcal{I}}$ may contain "unreachable" elements that are not needed to verify $d \in S^{\mathcal{I}}$. In this section we will show that, to decide satisfiability of feature terms, it suffices to consider only the roots of rooted feature algebras. This fact will be used in the next section to show that sort equations can be internalized.

Let $\mathcal{I}$ be a feature algebra and let $d$ be an element of $\mathbf{D}^{\mathcal{I}}$. We define

$$
g e n(d):=\left\{e \in \mathbf{D}^{\mathcal{I}} \mid \text { there exists a path } p \text { with } d p^{\mathcal{I}}=e\right\}
$$

and say that an element of $\operatorname{gen}(d)$ is generated by $d$. Obviously, $d \in \operatorname{gen}(d)$, and $e \in g e n(d)$ implies that $g e n(e) \subseteq g e n(d)$. 
Our intention is now to restrict the domains of feature algebras to such sets $\operatorname{gen}(d)$. However, we must keep in mind that atoms must always be interpreted somehow. Thus, if some elements of $\mathbf{A}^{\mathcal{I}}=\left\{a^{\mathcal{I}} \mid a \in \mathbf{A}\right\}$ are not contained in $g e n(d)$ we cannot really restrict the domain to $g e n(d)$, but only to $\operatorname{gen}(d) \cup \mathbf{A}^{\mathcal{I}}$.

We say that a feature algebra $\mathcal{I}$ is rooted iff there exists $d \in \mathrm{D}^{\mathcal{I}}$ such that $\mathbf{D}^{\mathcal{I}}=\operatorname{gen}(d) \cup \mathbf{A}^{\mathcal{I}}$. In this case, $d$ is called a root of $\mathcal{I}$.

In order to show that it is sufficient to consider such rooted feature algebras when interested in satisfiability of feature terms, we need the following weak notion of restriction of a feature algebra. Let $\mathcal{I}$ be a feature algebra and let $M$ be a subset of $\mathbf{D}^{\mathcal{I}}$. Then a feature algebra $\mathcal{J}$ is called a quasirestriction of $\mathcal{I}$ to the subset $M$ iff it satisfies the following properties:

1. $\mathbf{D}^{\mathcal{J}}=M \cup \mathbf{A}^{\mathcal{I}}$,

2. $a^{\mathcal{J}}=a^{\mathcal{I}}$ for all atoms $a$,

3. $A^{\mathcal{J}} \cap M=A^{\mathcal{I}} \cap M$ for all sorts $A$, and

4. $f^{\mathcal{J}}=f^{\mathcal{I}} \cap M \times M$ for all features $f$.

For a given feature algebra $\mathcal{I}$ and a subset $M$ of $\mathbf{D}^{\mathcal{I}}$ there may exist more than one quasi-restriction of $\mathcal{I}$ to $M$. These quasi-restrictions may differ in the behavior of elements of $\mathbf{A}^{\mathcal{I}}-M$ with respect to sorts. Nevertheless, we shall often use the name $\left.\mathcal{I}\right|_{M}$ for such a quasi-restriction. We call $\mathcal{J}$ quasirestriction of $\mathcal{I}$ and not restriction because usually one has that restrictions are unique. However, defining the notion "quasi-restriction to a set $M$ " in this non-unique way is necessary for the proof of Corollary 3.4, which in turn is important for the proofs of Lemma 4.1 and Theorem 4.4.

Lemma 3.1 Let $\left.\mathcal{I}\right|_{M}$ be a quasi-restriction of $\mathcal{I}$ to the subset $M$ of $\mathbf{D}^{\mathcal{I}}$. For all feature terms $S$ and all elements $d$ of $\mathbf{D}^{\mathcal{I}}$ satisfying gen $(d) \subseteq M$ we have

$$
d \in S^{\mathcal{I}} \Longleftrightarrow d \in S^{\left.\mathcal{I}\right|_{M}} .
$$

Proof. The lemma is proved by induction on the structure of $S$. Without loss of generality we may assume that $S$ contains only the forms $a, A, p \downarrow q$, $\exists L(T), T_{1} \sqcap T_{2}, \neg T$.

1. $S=a$ for an atom $a$. Since $\left.\mathcal{I}\right|_{M}$ is a quasi-restriction of $\mathcal{I}$ to $M$ we have $a^{\left.\mathcal{I}\right|_{M}}=a^{\mathcal{I}}$, and thus $d \in S^{\mathcal{I}}$ iff $d \in S^{\left.\mathcal{I}\right|_{M}}$ is trivially satisfied. 
2. $S=A$ for a sort $A$. We have $A^{\left.\mathcal{I}\right|_{M}} \cap M=A^{\mathcal{I}} \cap M$ since $\left.\mathcal{I}\right|_{M}$ is a quasi-restriction of $\mathcal{I}$ to $M$, and $d \in M$ since $g e n(d) \subseteq M$. This yields $d \in S^{\mathcal{I}}$ iff $d \in S^{\left.\mathcal{I}\right|_{M}}$.

3. $S=p \downarrow q$ for paths $p, q$. Let $p=f_{1} \ldots f_{k}$ and $q=g_{1} \ldots g_{l}$.

Assume that $d \in(p \downarrow q)^{\mathcal{I}}$, that is, $d p^{\mathcal{I}}$ and $d q^{\mathcal{I}}$ are both defined and equal. To be more precise, that means there exist $d_{1}, \ldots, d_{k}, e_{1}, \ldots$, $e_{l}$ in $\mathbf{D}^{\mathcal{I}}$ such that $\left(d, d_{1}\right) \in f_{1}^{\mathcal{I}},\left(d_{1}, d_{2}\right) \in f_{2}^{\mathcal{I}}, \ldots,\left(d_{k-1}, d_{k}\right) \in f_{k}^{\mathcal{I}}$, $\left(d, e_{1}\right) \in g_{1}^{\mathcal{I}},\left(e_{1}, e_{2}\right) \in g_{2}^{\mathcal{I}}, \ldots,\left(e_{l-1}, e_{l}\right) \in g_{l}^{\mathcal{I}}$, and $d_{k}=e_{l}$. Obviously, $d_{1}, \ldots, d_{k}, e_{1}, \ldots, e_{l}$ are all elements of $g e n(d)$, and thus of $M$. But then $d p^{\left.\mathcal{I}\right|_{M}}=d_{k}=e_{l}=d q^{\left.\mathcal{I}\right|_{M}}$, which shows $d \in(p \downarrow q)^{\left.\mathcal{I}\right|_{M}}$.

Conversely, assume that $d \in(p \downarrow q)^{\left.\mathcal{I}\right|_{M}}$, that is, $d p^{\left.\mathcal{I}\right|_{M}}=e=d q^{\left.\mathcal{I}\right|_{M}}$ for an element $e \in \mathbf{D}^{\left.\mathcal{I}\right|_{M}}$. Obviously, this implies $d p^{\mathcal{I}}=e=d q^{\mathcal{I}}$, and thus $d \in(p \downarrow q)^{\mathcal{I}}$.

4. $S=\exists L(T)$ for a feature term $T$ and a description $L$ of a regular set of paths.

Assume that $d \in(\exists L(T))^{\mathcal{I}}$, that is, there exists a path $p \in L$ and an element $e \in T^{\mathcal{I}}$ such that $d p^{\mathcal{I}}=e$. As above, $d p^{\mathcal{I}}=e$ implies $d p^{\left.\mathcal{I}\right|_{M}}=e$. In addition, $e \in \operatorname{gen}(d)$ yields $\operatorname{gen}(e) \subseteq \operatorname{gen}(d) \subseteq M$. Thus we can apply the induction hypothesis to $T$ and $e$, and get $e \in T^{\left.\mathcal{I}\right|_{M}}$. This shows $d \in(\exists L(T))^{\left.\mathcal{I}\right|_{M}}$.

The other direction can be proved in a similar way.

5. $S=T_{1} \sqcap T_{2}$. By induction, we have for $i=1,2$ that $d \in T_{i}^{\mathcal{I}}$ iff $d \in$ $T_{i}^{\left.\mathcal{I}\right|_{M}}$. This yields $d \in\left(T_{1} \sqcap T_{2}\right)^{\mathcal{I}}$ iff $d \in\left(T_{1} \sqcap T_{2}\right)^{\left.\mathcal{I}\right|_{M}}$.

6. $S=\neg T$. By induction, we have $d \in T^{\mathcal{I}}$ iff $d \in T^{\left.\mathcal{I}\right|_{M}}$. This yields $d \in(\neg T)^{\mathcal{I}}$ iff $d \in(\neg T)^{\left.\mathcal{I}\right|_{M}}$.

This completes the proof of the lemma.

If we take $M=g e n(d)$ in this lemma we get

Theorem 3.2 Let $\mathcal{I}$ be a feature algebra, $d$ be an element of $\mathbf{D}^{\mathcal{I}}$, and $S$ be a feature term. Then

$$
d \in S^{\mathcal{I}} \Longleftrightarrow d \in S^{\left.\mathcal{I}\right|_{g e n(d)}}
$$

provided that $\left.\mathcal{I}\right|_{\text {gen (d) }}$ is a quasi-restriction of $\mathcal{I}$ to gen $(d)$.

The theorem shows that one can restrict the attention to rooted feature algebras if one is interested in the satisfiability of a feature term. 
Corollary 3.3 A feature term $S$ is satisfiable if and only if there exists a rooted feature algebra $\mathcal{I}$ with root $d \in \mathbf{D}^{\mathcal{I}}$ such that $d \in S^{\mathcal{I}}$.

As another consequence of Theorem 3.2 one gets that the behavior of an atom with respect to feature terms only depends on its behavior with respect to sorts.

Corollary 3.4 Let $b$ be an atom, and let $\mathcal{I}$ and $\mathcal{J}$ be feature algebras such that $b^{\mathcal{I}} \in A^{\mathcal{I}}$ if and only if $b^{\mathcal{J}} \in A^{\mathcal{J}}$ holds for all sorts $A$. Then we have $b^{\mathcal{I}} \in S^{\mathcal{I}}$ if and only if $b^{\mathcal{J}} \in S^{\mathcal{J}}$ for all feature terms $S$.

Proof. For an atom $b$ the set $\operatorname{gen}\left(b^{\mathcal{I}}\right)$ is always a singleton set consisting of the element $b^{\mathcal{I}}$ alone. Thus any quasi-restriction $\mathcal{I}_{b}$ of a feature algebra $\mathcal{I}$ to $\operatorname{gen}\left(b^{\mathcal{I}}\right)$ has the set $\mathbf{A}^{\mathcal{I}}$ as its domain. By the definition of quasi-restrictions all the features are interpreted as empty relations in $\mathcal{I}_{b}$. For all sorts $A$ we have $b^{\mathcal{I}} \in A^{\mathcal{I}}$ iff $b^{\mathcal{I}_{b}} \in A^{\mathcal{I}_{b}}$, but the behavior of elements $a^{\mathcal{I}_{b}}$ for $a \neq b$ with respect to sorts is arbitrary. Together with Theorem 3.2 this observation completes the proof of the corollary.

As already pointed out earlier this corollary will be important for the proofs of Lemma 4.1 and Theorem 4.4.

\section{Internalizing Sort Equations}

As mentioned above it is sufficient to consider only one sort equation of the form $S \doteq \perp$. Recall that we denote by $\mathbf{F}^{*}$ the set of all paths, that is, the set of all words over $\mathbf{F}$.

Lemma 4.1 The feature term $T$ is satisfiable w.r.t. the sort equation $S \doteq \perp$ if and only if the feature term $T \sqcap \forall \mathbf{F}^{*}(\neg S)$, and the feature terms $\neg S \sqcap$ a for all atoms a are satisfiable.

Proof. Assume that $\mathcal{I}$ is a feature algebra such that $S^{\mathcal{I}}=\perp^{\mathcal{I}}=\emptyset$ and $T^{\mathcal{I}} \neq \emptyset$. Obviously, $S^{\mathcal{I}}=\emptyset$ means that all the elements of $\mathbf{D}^{\mathcal{I}}$ are in $(\neg S)^{\mathcal{I}}$. In particular, we have $a^{\mathcal{I}} \in(\neg S)^{\mathcal{I}}$ for all atoms $a$. This shows that $(\neg S \sqcap a)^{\mathcal{I}} \neq \emptyset$.

Let $d \in \mathbf{D}^{\mathcal{I}}$ be such that $d \in T^{\mathcal{I}}$. In order to prove that $d \in$ $\left(T \sqcap \forall \mathbf{F}^{*}(\neg S)\right)^{\mathcal{I}}$ it is enough to show that $d \in\left(\forall \mathbf{F}^{*}(\neg S)\right)^{\mathcal{I}}$. Let $p \in \mathbf{F}^{*}$ 
and $e \in \mathbf{D}^{\mathcal{I}}$ be such that $d p^{\mathcal{I}}=e$. Since all the elements of $\mathbf{D}^{\mathcal{I}}$ are in $(\neg S)^{\mathcal{I}}$ we have $e \in(\neg S)^{\mathcal{I}}$, which completes the proof of the "only-if" part of the lemma.

Conversely, assume that the feature term $T \sqcap \forall \mathbf{F}^{*}(\neg S)$ and the feature terms $\neg S \sqcap a$ for all atoms $a$ are satisfiable. Let $\mathcal{I}$ be a feature algebra such that $\left(T \sqcap \forall \mathbf{F}^{*}(\neg S)\right)^{\mathcal{I}} \neq \emptyset$, and for all atoms $a$ let $\mathcal{I}_{a}$ be a feature algebra such that $(\neg S \sqcap a)^{\mathcal{I}} \neq \emptyset$. Let $d \in \mathbf{D}^{\mathcal{I}}$ be such that $d \in\left(T \sqcap \forall \mathbf{F}^{*}(\neg S)\right)^{\mathcal{I}}$. We want to define a quasi-restriction $\left.\mathcal{I}\right|_{g e n(d)}$ of $\mathcal{I}$ to $g e n(d)$ which satisfies the sort equation $S \doteq \perp$ and interprets the feature term $T$ as nonempty set. For that purpose we have to fix the interpretation of the sorts on $R A:=\mathbf{A}^{\mathcal{I}}-g e n(d)$ in an appropriate way. This can be done as follows: For all sorts $A$ we define

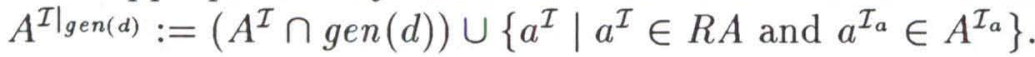

By Theorem 3.2 we have $d \in\left(T \sqcap \forall \mathbf{F}^{*}(\neg S)\right)^{\left.\mathcal{I}\right|_{g e n(d)}}$ since $d \in$ $\left(T \sqcap \forall \mathbf{F}^{*}(\neg S)\right)^{\mathcal{I}}$. In particular, this yields $d \in T^{\left.\mathcal{I}\right|_{g e n(d)}}$. It remains to be shown that $S^{\left.\mathcal{I}\right|_{\text {gen(d) }}}=\emptyset$. Assume that there exists $e \in \mathbf{D}^{\left.\mathcal{I}\right|_{g e n(d)}}=g e n(d) \cup R A$ such that $e \in S^{\left.\mathcal{I}\right|_{g e n(d)}}$.

If $e \in \operatorname{gen}(d)$ then there exist a path $p \in \mathbf{F}^{*}$ such that $e=d p^{\mathcal{I}}$. But then

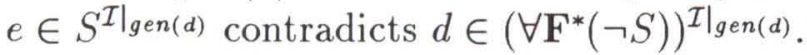

Assume that $e \in R A$, that is, $e=a^{\mathcal{I}}$ for an atom $a$ such that $a^{\mathcal{I}} \notin g e n(d)$.

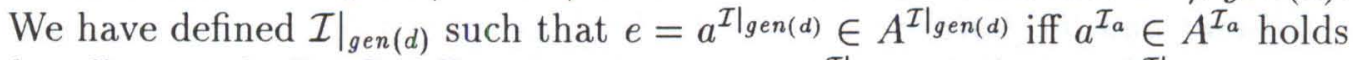
for all sorts $A$. By Corollary 3.4 we get $e=a^{\left.\mathcal{I}\right|_{g e n(d)}} \in(\neg S \sqcap a)^{\left.\mathcal{I}\right|_{g e n(d)}}$ since

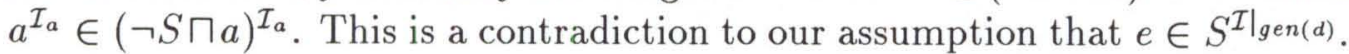

Let us now consider the following condition more closely.

Condition 4.2 For all atoms a the feature term $\neg S \sqcap a$ is satisfiable.

If there are no atoms, that is, if $\mathbf{A}=\emptyset$, then this condition is void. This yields

Theorem 4.3 Assume that $\mathbf{A}=\emptyset$. Then the feature term $T$ is satisfiable w.r.t. the sort equation $S \doteq \perp$ if and only if the feature term $T \sqcap \forall \mathbf{F}^{*}(\neg S)$ is satisfiable.

Now assume that there exists at least one atom. In this case, Condition 4.2 characterizes the consistency of the sort equation $S \doteq \perp$. 
Theorem 4.4 Assume that $\mathbf{A} \neq \emptyset$. Then the sort equation $S \doteq \perp$ is consistent if and only if for all atoms a the feature term $\neg S \sqcap a$ is satisfiable.

Proof. Let $\mathcal{I}$ be a model of the sort equation $S \doteq \perp$. This means that $S^{\mathcal{I}}=\emptyset$, and thus $(\neg S)^{\mathcal{I}}=\mathbf{D}^{\mathcal{I}}$. Consequently, we have for any atom $a$ that $a^{\mathcal{I}} \in \mathbf{D}^{\mathcal{I}}=(\neg S)^{\mathcal{I}}$. But then $a^{\mathcal{I}} \in(\neg S \sqcap a)^{\mathcal{I}}$, which shows that this feature term is satisfiable.

On the other hand, assume that for any atom $a$ there is a feature algebra $\mathcal{I}_{a}$ such that $(\neg S \sqcap a)^{\mathcal{I}_{a}} \neq \emptyset$. This means that for any atom $a$ we have $a^{\mathcal{I}_{a}} \in(\neg S)^{\mathcal{I}_{a}}$. We define a new feature algebra $\mathcal{I}$ as follows: $\mathbf{D}^{\mathcal{I}}:=\left\{a^{\mathcal{I}} \mid\right.$ $a$ is an atom $\}$ where the $a^{\mathcal{I}}$ are assumed to be different individuals; for all features $f$ we define $f^{\mathcal{I}}:=\emptyset$; and for all sorts $A$ we define $A^{\mathcal{I}}:=\left\{a^{\mathcal{I}} \mid a^{\mathcal{I}_{a}} \in\right.$ $\left.A^{\mathcal{I}_{a}}\right\}$. Obviously, $\mathcal{I}$ is a feature algebra. By Corollary 3.4 we get for all atoms $a$ that $a^{\mathcal{I}} \in(\neg S)^{\mathcal{I}}$ because $a^{\mathcal{I}_{a}} \in(\neg S)^{\mathcal{I}_{a}}$. This shows that $(\neg S)^{\mathcal{I}}=\mathbf{D}^{\mathcal{I}}$, and thus $S^{\mathcal{I}}=\emptyset$.

This theorem together with the lemma yields

Theorem 4.5 Assume that $\mathbf{A} \neq \emptyset$. Then the feature term $T$ is satisfiable w.r.t. the sort equation $S \doteq \perp$ if and only if the sort equation $S \doteq \perp$ is consistent and the feature term $T \sqcap \forall \mathbf{F}^{*}(\neg S)$ is satisfiable.

\section{Consistency of Sort Equations}

Assume that $\mathbf{A} \neq \emptyset$, that is, there is at least one atom. Then Theorem 4.4 yields a decision criterion for consistency of sort equations of the form $S \doteq \perp$, and thus for consistency of arbitrary finite sets of sort equations.

Theorem 5.1 Assume that $\mathbf{A} \neq \emptyset$, and let $\mathcal{E}$ be a finite set of sort equations. Then it is decidable whether $\mathcal{E}$ is consistent or not.

Proof. The first problem is that, if the set of atoms is infinite, we should have to consider infinitely many terms of the form $\neg S \sqcap a$ in order to check Condition 4.2. However, the sort equations contain only finitely many atoms. It is easy to see that it is enough to consider these finitely many atoms, and only one of the other atoms as specimen. Thus it remains to be shown that one can decide satisfiability of feature terms of the form $\neg S \sqcap a$. But this is an easy consequence of the next lemma since satisfiability of feature terms not containing path quantifications is decidable [20]. 
Lemma 5.2 Let $T$ be a feature term and $a$ be an atom. Then there exists a feature term $T^{\prime}$ without path quantifications such that $T \sqcap a \sim T^{\prime} \sqcap a$.

Proof. It is easy to see that a term of the form $\forall L\left(T_{1}\right) \sqcap a$ or $\exists L\left(T_{1}\right) \sqcap a$ is equivalent to the term $T_{1} \sqcap a$ if the empty word $\varepsilon$ is in $L$. If $\varepsilon \notin L$, then $\forall L\left(T_{1}\right) \sqcap a$ is equivalent to $\top \sqcap a$ and $\exists L\left(T_{1}\right) \sqcap a$ is equivalent to $\perp \sqcap a$. Using this fact, the lemma can easily be proved by induction. Please note that, if $T$ starts with a negation, then this negation can be pushed into the term with the help of de Morgan's rules and the fact that $\neg \forall L(S) \sim \exists L(\neg S)$ and $\neg \exists L(S) \sim \forall L(\neg S)$.

If $\mathbf{A}=\emptyset$, that is, there is no atom, then consistency of sort equations may become undecidable, even if the terms occurring in the sort equations do not contain path quantifications. We shall prove this result by a reduction of the word problem for groups. To this purpose we rephrase the word problem in such a way that it fits into our framework.

Let $\Sigma$ be a nonempty set of symbols, $\Sigma^{*}$ be the set of words over $\Sigma$, and $\varepsilon$ be the empty word. Under concatenation of words, $\Sigma^{*}$ is a monoid whose neutral element is $\varepsilon$. A congruence is an equivalence relation $\sim$ on $\Sigma^{*}$ such that $p \sim q$ implies $r p r^{\prime} \sim r q r^{\prime}$ for all $p, q, r, r^{\prime} \in \Sigma^{*}$. If $\sim$ is clear from the context, we use $\bar{p}$ to denote the equivalence class of a word $p \in \Sigma^{*}$ with respect to $\sim$. The quotient $\Sigma^{*} / \sim$ is again a monoid under the operation $\bar{p} \bar{q}:=\overline{p q}$.

A Thue equation over $\Sigma$ is a set $\{p, q\}$ consisting of two words $p, q \in \Sigma^{*}$. A Thue system over $\Sigma$ is a finite set $T$ of Thue equations over $\Sigma$. Every Thue system $T$ over $\Sigma$ defines a binary relation $\leftrightarrow_{T}$ on $\Sigma^{*}$ by

$$
u \leftrightarrow_{T} v: \Longleftrightarrow \exists w_{1}, w_{2} \in \Sigma^{*} \exists\{p, q\} \in T: \quad u=w_{1} p w_{2} \wedge v=w_{1} q w_{2} .
$$

We use $\sim_{T}$ to denote the reflexive and transitive closure of $\leftrightarrow_{T}$ on $\Sigma^{*}$. It is easy to see that $\sim_{T}$ is a congruence on $\Sigma^{*}$. To be more precise, $\sim_{T}$ is the least congruence $\sim$ such that $p \sim q$ for every Thue equation $\{p, q\}$ in $T$.

It is known that there exists a Thue system $T$ consisting of seven equations over a two-element alphabet such that it is undecidable for two words $p, q$ whether $p \sim_{T} q$ holds or not (see, for instance, [2]). In the following we shall need a stronger version of this undecidability result, which is due to Novikov and Boone (see $[13,2,21])$ : there is a finite set of symbols $\Sigma$ and a Thue system $G=\left\{\left\{p_{1}, \varepsilon\right\}, \ldots,\left\{p_{n}, \varepsilon\right\}\right\}$ such that

1. for every $f \in \Sigma$ there is some $q \in \Sigma^{*}$ such that $G$ contains the Thue equation $\{f q, \varepsilon\}$ 
2. the set of words $p$ with $p \chi_{G} \varepsilon$ is not recursively enumerable.

In particular, it is undecidable whether $p \sim_{G} \varepsilon$ or not. Note that property (1) implies that $\Sigma^{*} / \sim_{G}$ is a group.

Theorem 5.3 Assume that $\mathbf{A}=\emptyset$, that is, there is no atom. Then there exists a feature term $S$ of the form

$$
p_{1} \downarrow \varepsilon \sqcap \ldots \sqcap p_{n} \downarrow \varepsilon
$$

such that the set of paths $p$ for which the sort equation

$$
S \sqcap \neg(p \downarrow \varepsilon) \doteq \mathrm{T}
$$

is consistent is not recursively enumerable. In particular, it is undecidable whether the sort equation $S \sqcap \neg(p \downarrow \varepsilon) \doteq \top$ is consistent or not.

Proof. Suppose that $\Sigma$ is a set of symbols and $G=\left\{\left\{p_{1}, \varepsilon\right\}, \ldots,\left\{p_{n}, \varepsilon\right\}\right\}$ a Thue system over $\Sigma$ with the properties stated in the theorem by Novikov and Boone. We regard elements of $\Sigma$ as features and words over $\Sigma$ as paths. Let $S$ be the feature term

$$
p_{1} \downarrow \varepsilon \sqcap \ldots \sqcap p_{n} \downarrow \varepsilon
$$

To prove our claim it suffices to show that for every $p \in \Sigma^{*}$ the sort equation $S \sqcap \neg(p \downarrow \varepsilon) \doteq \top$ is consistent if and only if $p \varkappa_{G} \varepsilon$.

“ $\Rightarrow$ Suppose $p \chi_{G} \varepsilon$. We construct a feature algebra $\mathcal{I}$ satisfying $S \sqcap \neg(p \downarrow \varepsilon) \doteq \top$ as follows:

$$
\begin{aligned}
\mathbf{D}^{\mathcal{I}} & :=\Sigma^{*} / \sim_{G} \\
\bar{q} f^{\mathcal{I}} & :=\overline{q f} \text { for every } f \in \Sigma \text { and } q \in \Sigma^{*} .
\end{aligned}
$$

Since $\sim_{G}$ is the congruence generated by $G$, we have $\bar{p}_{i}=\bar{\varepsilon}$ for every Thue equation $\left\{p_{i}, \varepsilon\right\}$ in $G$. This implies $\bar{q} p_{i}^{\mathcal{I}}=\overline{q p_{i}}=\overline{q \varepsilon}=\bar{q}$ for every $\bar{q} \in \mathbf{D}^{\mathcal{I}}$. Hence, $\mathcal{I}$ satisfies $S \doteq \mathrm{T}$.

Assume that $\mathcal{I}$ does not satisfy $\neg(p \downarrow \varepsilon) \doteq \mathrm{T}$. Then there is some $\bar{q} \in \mathbf{D}^{\mathcal{I}}$ such that $\bar{q} p^{\mathcal{I}}=\bar{q}$, which implies that $\bar{q}=\overline{q p}=\bar{q} \bar{p}$. Since $\Sigma^{*} / \sim_{G}$ is a group, the element $\bar{q}$ has an inverse $\overline{q^{\prime}}$. Then $\bar{p}=\overline{q^{\prime}} \bar{q} \bar{p}=\overline{q^{\prime}} \bar{q}=\bar{\varepsilon}$, that is $p \sim_{G} \varepsilon$. We thus have obtained a contradiction to the fact that $p \psi_{G} \varepsilon$. Hence, $\bar{q} p^{\mathcal{I}} \neq \bar{q}$ for all $\bar{q} \in \mathbf{D}^{\mathcal{I}}$, which implies that $\mathcal{I}$ satisfies $\neg(p \downarrow \varepsilon) \doteq \mathrm{T}$. Since $\mathcal{I}$ satisfies $S \doteq \top$ and $\neg(p \downarrow \varepsilon) \doteq \top$, it follows that $\mathcal{I}$ satisfies $S \sqcap \neg(p \downarrow \varepsilon) \doteq \top$. 
"६" Suppose $p \sim_{G} \varepsilon$. Assume there is a feature algebra $\mathcal{I}$ that satisfies $S \sqcap \neg(p \downarrow \varepsilon) \doteq \top$. We define an equivalence relation $\sim$ on $\Sigma^{*}$ by

$$
q \sim q^{\prime} \quad: \Longleftrightarrow \mathcal{I} \text { satisfies } q \downarrow q^{\prime} \doteq \top .
$$

Since $\mathcal{I}$ satisfies $S \sqcap \neg(p \downarrow \varepsilon) \doteq \top$, it follows that $\mathcal{I}$ satisfies $p_{i} \downarrow \varepsilon \doteq \top$ for every Thue equation $\left\{p_{i}, \varepsilon\right\}$ in $G$. By property (1) of $G$, for every $f \in \Sigma$ there is some $q \in \Sigma^{*}$ such that $\mathcal{I}$ satisfies $f q \downarrow \varepsilon \doteq \mathrm{T}$. This means that for every $d \in \mathbf{D}^{\mathcal{I}}$ we have $d f^{\mathcal{I}} q^{\mathcal{I}}=d$. Hence, every $f^{\mathcal{I}}$ is a total function on $\mathbf{D}^{\mathcal{I}}$. We conclude that for all $q, q^{\prime}, r, r^{\prime} \in \Sigma^{*}$ the feature algebra $\mathcal{I}$ satisfies $r q r^{\prime} \downarrow r q^{\prime} r^{\prime} \doteq \mathrm{T}$ if $\mathcal{I}$ satisfies $q \downarrow q^{\prime} \doteq \mathrm{T}$. Thus, $\sim$ is a congruence.

For every Thue equation $\left\{p_{i}, \varepsilon\right\}$ in $G$, the feature algebra $\mathcal{I}$ satisfies $p_{i} \downarrow \varepsilon \doteq \top$, which implies $p_{i} \sim \varepsilon$. By definition, $\sim_{G}$ is the least congruence with this property. Therefore, $p \sim_{G} \varepsilon$ implies $p \sim \varepsilon$, that is, $\mathcal{I}$ satisfies $p \downarrow \varepsilon \doteq \top$. This contradicts our assumption that $\mathcal{I}$ satisfies $\neg(p \downarrow \varepsilon) \doteq T$. We conclude that $S \sqcap \neg(p \downarrow \varepsilon) \doteq \top$ is unsatisfiable.

\section{Satisfiability is Undecidable}

As an easy consequence of Theorem 5.3 we get that satisfiability of feature terms w.r.t. sort equations is undecidable, if we have no atoms. In fact, the feature term $T$ is satisfiable w.r.t. a finite set of sort equations $\mathcal{E}$ if and only if $\mathcal{E}$ is consistent.

We have seen that consistency of sort equations is decidable, if we have at least one atom. But satisfiability of feature terms w.r.t. sort equations is nevertheless undecidable in this case. This is shown in [20] in the presence of three features, two atoms and one sort. The sort equations and the feature term constructed in [20] do not contain path quantifications.

Taking the two results together we thus have

Theorem 6.1 Satisfiability of feature terms w.r.t. sort equations is undecidable. This holds even if path quantifications are disallowed, and it does not depend on whether $\mathbf{A}=\emptyset$ or $\mathbf{A} \neq \emptyset$.

In the light of Section 4, this theorem shows that satisfiability of feature terms with path quantifications is undecidable, independently on whether we have atoms or not. 
Theorem 6.2 Satisfiability of feature terms with path quantification is undecidable. This result does not depend on whether $\mathbf{A}=\emptyset$ or $\mathbf{A} \neq \emptyset$.

Proof. Assume that satisfiability of feature terms with path quantifications is decidable. Then the characterizations of satisfiability of feature terms w.r.t. sort equations given in Theorem 4.3 (for $\mathbf{A}=\emptyset$ ) or Theorem 4.5 (for $\mathbf{A} \neq \emptyset$ ) would yield a decision criterion for satisfiability w.r.t. sort equations. This is a contradiction to Theorem 6.1.

\section{Conclusion}

We have studied the expressivity of functional uncertainty in a feature term language with negation and obtained two main results: satisfiability is undecidable and sort equations can be internalized. For practical applications in grammar formalisms this language is probably too expressive since general negation is not needed. Thus it would be interesting to find out whether satisfiability of feature terms built from the forms $a, A, p \downarrow q, \exists L(S)$, and $S \sqcap S^{\prime}$ is decidable.

Feature logics are closely related to terminological logics $[3,11,12,17]$, which are employed in knowledge representation and grew out of research in semantic networks and frame systems. The essential difference between these two formalisms is that in terminological logics attributes can be nonfunctional while they must be functional in feature logics.

Baader [1] studies a terminological logic that can be obtained from the feature logic in this paper by three changes: disallow atoms and agreements, and admit also interpretations that interpret features as nonfunctional binary relations. He shows that in this logic satisfiability of "feature terms" (which are called concept terms in this context) is decidable. Since concept equations (i.e., the equivalent of the sort equations of the present paper) can also be internalized with the help of path quantifications, the algorithm given in [1] also yields a decision procedure for satisfiability w.r.t. concept equations. Baader's algorithm can easily be adapted to the case where one allows only functional binary relations. This means that the feature logic of the present paper becomes decidable if agreements and atoms are disallowed.

Similar results for terminological logics have independently been obtained by Schild [16] as byproducts of the correspondence he exhibits between terminological logics and dynamic logics. In addition, he shows that this correspondence also yields complexity results for the terminological logic considered 
by Baader, and for our feature logic if agreements and atoms are disallowed. In both cases, one has an EXPTIME-complete satisfiability problem.

\section{References}

[1] F. Baader. Augmenting concept languages by transitive closure of roles: An alternative to terminological cycles. Research Report RR-90-13, DFKI, 6750 Kaiserslautern, Germany, 1990.

[2] W. W. Boone. The word problem. Ann. of Mat., 70:207-265, 1959.

[3] R. J. Brachman and J. G. Schmolze. An overview of the KL-ONE knowledge representation system. Cognitive Science, 9(2):171-216, Apr. 1985 .

[4] J. Dörre and W. C. Rounds. On subsumption and semi-unification in feature algebras. In Proceedings of the 5th Annual IEEE Symposium on Logic in Computer Science, pages 301-310, Philadelphia, PA, 1990.

[5] M. Johnson. Attribute-Value Logic and the Theory of Grammar. CSLI Lecture Notes 16. Center for the Study of Language and Information, Stanford University, Cal., 1988.

[6] R. M. Kaplan and J. Bresnan. Lexical-Functional Grammar: A formal system for grammatical representation. In J. Bresnan, editor, The Mental Representation of Grammatical Relations, pages 173-381. MIT Press, Cambridge, Mass., 1982.

[7] R. M. Kaplan and J. T. Maxwell III. An algorithm for functional uncertainty. In Proceedings of the 12th International Conference on Computational Linguistics, pages 297-302, Budapest, Hungary, 1989.

[8] R. M. Kaplan and J. T. Maxwell III. Constituent coordination in lexicalfunctional grammar. In Proceedings of the 12th International Conference on Computational Linguistics, pages 303-305, Budapest, Hungary, 1989.

[9] R. T. Kasper and W. C. Rounds. A logical semantics for feature structures. In Proceedings of the 24th Annual Meeting of the ACL, Columbia University, pages 257-265, New York, N.Y., 1986.

[10] M. Kay. Functional grammar. In Proceedings of the Fifth Annual Meeting of the Berkeley Linguistics Society, Berkeley, Cal., 1979. Berkeley Linguistics Society. 
[11] H. J. Levesque and R. J. Brachman. Expressiveness and tractability in knowledge representation and reasoning. Computational Intelligence, 3:78-93, 1987.

[12] B. Nebel and G. Smolka. Representation and reasoning with attributive descriptions. In K. Bläsius, U.Hedtstück, and C.-R. Rollinger, editors, Sorts and Types in Artificial Intelligence, volume 418 of Lectures Notes in Computer Science, pages 112-139. Springer-Verlag, Berlin, Germany, 1990 .

[13] P. S. Novikov. On the algorithmic unsolvability of the word problem (in Russian). Dokl. Akad. Nauk SSSR Mathematičeskii Institut Trudy, 44, 1955. Moscow.

[14] C. Pollard and I. A. Sag. Information-Based Syntax and Semantics, volume 13 of CSLI Lecture Notes. Center for the Study of Language and Information, Stanford University, Cal., 1987.

[15] W. C. Rounds and R. T. Kasper. A complete logical calculus for record structures representing linguistic information. In Proceedings of the 1st IEEE Symposium on Logic in Computer Science, pages 38-43, Boston, Mass., 1986.

[16] K. Schild. A correspondence theory for terminological logics: Preliminary report. Preprint, TU Berlin, Project KIT-BACK, 1000 Berlin, Germany, 1990.

[17] M. Schmidt-Schauß and G. Smolka. Attributive concept descriptions with complements. Artificial Intelligence, (47), 1991.

[18] S. M. Shieber. An Introduction to Unification-Based Approaches to Grammar, volume 4 of CSLI Lecture Notes. Center for the Study of Language and Information, Stanford University, Cal., 1986.

[19] S. M. Shieber, H. Uszkoreit, F. C. Pereira, J. Robinson, and M. Tyson. The formalism and implementation of PATR-II. In J. Bresnan, editor, Research on Interactive Acquisition and Use of Knowledge. SRI International, Artificial Intelligence Center, Menlo Park, Cal., 1983.

[20] G. Smolka. A feature logic with subsorts. LILOG Report 33, IWBS, IBM Deutschland, Postfach 8008 80, 7000 Stuttgart 80, Germany, May 1988.

[21] G. Smolka. Feature constraint logics for unification grammars. IWBS Report 93, IWBS, IBM Deutschland, Postfach 8008 80, 7000 Stuttgart 
80, Germany, November 1989. To appear in the Journal of Logic Programming.

[22] J. Stillwell. The word problem and the isomorphism problem for groups. Bulletin AMS, 6:33-56, 1982. 


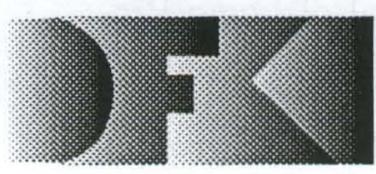

Deutsches

Forschungszentrum

für Künstliche

Intelligenz GmbH

\section{DFKI Publikationen}

Die folgenden DFKI Veröffentlichungen oder die aktuelle Liste von erhältlichen Publikationen können bezogen werden von der oben angegebenen Adresse.
DFKI

-Bibliothek-

Stuhlsatzenhausweg 3

6600 Saarbrücken 11

FRG

\section{DFKI Research Reports}

RR-90-01

Franz Baader

Terminological Cycles in KL-ONE-based

Knowledge Representation Languages

33 pages

RR-90-02

Hans-Jürgen Bürckert

A Resolution Principle for Clauses with

Constraints

25 pages

RR-90-03

Andreas Dengel \& Nelson M. Mattos

Integration of Document Representation,

Processing and Management

18 pages

RR-90-04

Bernhard Hollunder \& Werner Nutt

Subsumption Algorithms for Concept

Languages

34 pages

RR-90-05

Franz Baader

A Formal Definition for the Expressive

Power of Knowledge Representation

Languages

22 pages

RR-90-06

Bernhard Hollunder

Hybrid Inferences in KL-ONE-based

Knowledge Representation Systems

21 pages
RR-90-07

\section{Elisabeth André, Thomas Rist}

Wissensbasierte Informationspräsentation: Zwei Beiträge zum Fachgespräch Graphik und $\mathrm{KI}$ :

1. Ein planbasierter Ansatz zur Synthese illustrierter Dokumente

2. Wissensbasierte Perspektivenwahl für die automatische Erzeugung von 3DObjektdarstellungen

24 pages

RR-90-08

Andreas Dengel

A Step Towards Understanding Paper

Documents

25 pages

RR-90-09

Susanne Biundo

Plan Generation Using a Method of

Deductive Program Synthesis

17 pages

RR-90 - 10

Franz Baader, Hans-Jürgen Bürckert,

Bernhard Hollunder, Werner Nutt, Jörg H.

Siekmann

Concept Logics

26 pages

RR-90-11

Elisabeth André, Thomas Rist

Towards a Plan-Based Synthesis of

Illustrated Documents

14 pages 
R R-90-12

Harold Boley

Declarative Operations on Nets

43 pages

R R-90-13

Franz Baader

Augmenting Concept Languages by

Transitive Closure of Roles: An Alternative

to Terminological Cycles

40 pages

R R-90-14

Franz Schmalhofer, Otto Kühn, Gabriele

Schmidt

Integrated Knowledge Acquisition from

Text, Previously Solved Cases, and Expert

Memories

20 pages

R R-90-15

Harald Trost

The Application of Two-level Morphology to Non-concatenative German Morphology

13 pages

R R-90- 16

Franz Baader, Werner Nutt

Adding Homomorphisms to

Commutative/Monoidal Theories, or:

How Algebra Can Help in Equational

Unification

25 pages

R R-91-01

Franz Baader, Hans-Jürgen Bürckert,

Bernhard Nebel, Werner Nutt, and

Gert Smolka

On the Expressivity of Feature Logics with

Negation, Functional Uncertainty, and Sort

Equations

20 pages

\section{DFKI Technical Memos}

TM-89-01

Susan Holbach-Weber

Connectionist Models and Figurative

Speech

27 pages

TM-90-01

Som Bandyopadhyay

Towards an Understanding of Coherence in Multimodal Discourse

18 pages
TM-90-02

Jay C. Weber

The Myth of Domain-Independent

Persistence

18 pages

TM-90-03

Franz Baader, Bernhard Hollunder

KRIS: Knowledge Representation and

Inference System

-System Description-

15 pages

TM-90-04

Franz Baader, Hans-Jürgen Bürckert, Jochen Heinsohn, Bernhard Hollunder, Jürgen Müller, Bernhard Nebel, Werner Nutt, Hans-Jürgen Profitlich

Terminological Knowledge Representation: A Proposal for a Terminological Logic 7 pages

\section{DFKI Documents}

\section{D-89-01}

Michael H. Malburg \& Rainer Bleisinger

HYPERBIS: ein betriebliches HypermediaInformationssystem

43 Seiten

D-90-01

DFKI Wissenschaftlich-Technischer

Jahresbericht 1989

45 pages

D-90-02

Georg Seul

Logisches Programmieren mit Feature -

Typen

107 Seiten

D-90-03

Ansgar Bernardi, Christoph Klauck, Ralf Legleitner

Abschlußbericht des Arbeitspaketes PROD

36 Seiten

D-90-04

Ansgar Bernardi, Christoph Klauck, Ralf

Legleitner

STEP: Überblick über eine zukünftige Schnittstelle zum Produktdatenaustausch 69 Seiten 


\section{D-90-05}

Ansgar Bernardi, Christoph Klauck, Ralf

Legleitner

Formalismus zur Repräsentation von Geometrie- und Technologieinformationen als

Teil eines Wissensbasierten Produktmodells 66 Seiten

D-90-06

Andreas Becker

The Window Tool Kit

66 Seiten 



Franz Baader, Hans-Jürgen Bürckert, Bernhard Nebel, Werner Nutt, and Gert Smolka 